\title{
Gestión del conocimiento en las facultades de medicina. Estrategia de control de las enfermedades crónicas no transmisibles
}

\author{
Santos Depine ${ }^{1}$ \\ 1 Grupo de Investigación de Nefrología, Universidad Simón Bolívar. Barranquilla, Colombia. Calidad y \\ Salud Renal, Confederación de Asociaciones de Diálisis de la República Argentina.
}

\section{Resumen}

El mundo actual, está sometido a cambios permanentes, profundos y rápidos. La tecnología avanza a un ritmo antes impensado, proveyendo herramientas de transferencia y globalización de la información y poniendo a disposición del público en general el conocimiento producido.

Sin embargo, no existe una adecuada gestión de ese conocimiento en la mayoría de los espacios de educación superior, siendo las universidades meras transmisoras de conocimiento, enfocándose en el explícito y sin darle un lugar de importancia al conocimiento tácito, para gestionarlo y transformarlo.

Los cambios que se están dando en el proceso de envejecimiento, los impactos del medio ambiente, el crecimiento acrítico de las grandes ciudades, el sedentarismo (muchas veces asociado a la falta de espacios saludables y a la inseguridad), están modificando el mapa de las enfermedades, potenciando la aparición y persistencia de enfermedades degenerativas.

Estas enfermedades crónicas no transmisibles, tales como las cardiovasculares, diabetes y la insuficiencia renal, se han establecido en niveles de epidemia, sin que la enseñanza de la medicina se haya modificado en consonancia con las nuevas necesidades.

En las facultades de medicina es imprescindible generar proyectos innovadores, que impliquen cambios paradigmáticos curriculares, enfatizando los conceptos de salud como un todo, más que el de la enfermedad y sus tratamientos.

Este proyecto, presenta un modelo integrado para implementar la Gestión del Conocimiento en los espacios intra y extramurales de la universidad, con el objetivo de potenciar sociedades de conocimiento, articulando acciones entre docentes y alumnos, universidad y comunidad, con el objetivo de generar graduados que puedan satisfacer las demandas reales de la sociedad.

Palabras clave: Gestión del conocimiento, educación superior, enfermedades crónicas, atención primaria.

\section{Knowledge management in the schools of medicine. Control strategy for noncommuni-} cable diseases (NCDs)

\section{Summary}

Today the world is experiencing changes permanent, deep and fast. Technology is advancing at an unthinkable pace before, providing tools for transfer and the globalization of information of the new knowledge.

However, is poor the management of that knowledge in areas of higher education. Universities are only transmitters of knowledge, focusing on explicit knowledge and ignoring the tacit knowledge to transform it. 
The changes that are occurring in the process of aging of the population, the environmental impacts, the uncritical growth of large cities, the sedentary lifestyle (often associated with lack of health and insecurity), are changing the map of diseases, favoring the emergence and persistence of degenerative diseases.

These noncommunicable diseases such as cardiovascular disease, diabetes and renal failure have been established at epidemics levels without the necessary change in teaching of medicine in line with the new requirements.

In medical schools is essential generate innovative projects involving curriculum and paradigms shifts, highlighting the concepts of holistic health rather than the disease and its treatments.

This project presents an integrated model for the knowledge management in the intramural and extramural areas of the University, with the aim of generate knowledge societies and strengthen the coordination of activities between teachers and students, the University and the community, with the aim of creating graduates who can meet the actual demands of the society.

Key words: Knowledge Management. Higher education. Chronic diseases. Primary care.

\section{Introducción}

A ctualmente, el mundo y sus organizaciones viven en situación de cambio permanente. La rapidez y accesibilidad de las comunicaciones y las nuevas tecnologías de información, han impactado en la creación y en la duplicación del conocimiento, muchas veces sin demasiados "filtros" que aseguren la calidad de los mismos.

Ese aspecto es de enorme importancia, porque el conocimiento, en sí mismo, genera un valor competitivo para quien lo posee y es también un vehículo de aprendizaje, en el proceso de desaprender para aprender.

Conocimiento, no es lo mismo que aprendizaje o información. Es un activo intangible cuya posesión genera para quien lo posee una ventaja competitiva.

Nonaka y Takeuchi (1995) definieron el conocimiento como "un proceso humano y dinámico de justificar las creencias personales en busca de la verdad". Se sugiere que el conocimiento es dependiente del contexto ${ }^{1}$.

La Gestión del Conocimiento se transformó en una herramienta fundamental para el logro de resultados, ya sea en los ámbitos organizacionales, empresariales, en la investigación y en la docencia.

Tuvo sus orígenes en el ámbito empresarial, durante la década de los 90 en pleno auge de la globalización del conocimiento y de la economía a escala mundial, siendo posterior su incorporación a las universidades.

Se ha mencionado ${ }^{2}$ que la extensión de esta nueva cultura organizacional a diversos ámbitos tiene que ver con el despliegue de la "sociedad del conocimiento", anunciada por Peter Drucker en "Las nuevas realidades"3.

Existen empresas, que se involucran en proyectos de investigación y de innovación tecnológica, siendo productoras, administradoras y transferidoras de conocimientos. Ejemplo de ello, son las empresas farmacéuticas, cuyo impacto en los procesos de investigación de nuevas tecnologías, ha llevado a una mejora sustancial de la calidad de vida de las personas.

Las empresas gestionan recursos humanos y materiales de manera inteligente mientras que las universidades se desarrollan en función de "modelos de conocimiento", a su propio currículo y al perfil de los docentes, por su forma de gobierno y su relación con la sociedad.

El trabajo académico está organizado en torno a materiales cuya naturaleza es singularmente intelectual $^{4}$ y si bien los autores clásicos ya habían escrito acerca del valor de las personas en las organizaciones, la Gestión del Conocimiento implica un cambio paradigmático, por el cual ellas priorizan la educación de sus miembros, propendiendo a la creatividad, a la innovación y al pensamiento sistémico ${ }^{5}$. 
A partir de cómo se gestiona ese conocimiento, desde su creación, su distribución, su enseñanza y su aplicación práctica, se podrán alcanzar resultados extraordinarios o, por el contrario, no lograr los objetivos propuestos.

Gestionar el conocimiento implica agregarle valor, partiendo del conocimiento previo, la experiencia acumulada por su uso, estableciendo una espiral ascendente de generación de nuevo conocimiento que posibilite el logro de ventajas estratégicas sustentables y sostenibles en los procesos o programas desarrollados. Debe tomar lo general y llevarlo, en su proceso de gestión, a lo particular y al empoderamiento de cada persona de una organización para que sea un conocimiento aprehendido.

El rol de las universidades en este novedoso proceso de Gestión del Conocimiento resulta fundamental, en la formación de profesionales calificados, en sus docentes, sus investigadores y en los programas de extensión universitaria, con el objetivo de dar respuesta a las necesidades de la sociedad con la que interactúa, propiciando verdaderas sociedades del conocimiento.

De nada sirve poseer fuertes áreas de investigación que generen conocimientos, si no se trasladan al cuerpo académico de la universidad $\mathrm{y}$, por ende, a los alumnos. Es la diferencia de tener capital intelectual individual o consolidar el capital intelectual colectivo.

Es imprescindible esforzarse en lograr acreditadas áreas de investigación, cuyos logros deben culminar en procesos de innovación y nuevas maneras de ver el mundo en cualquiera de las competencias en las que el profesional se desarrolle, luego de su paso por la universidad.

En los últimos años, se ha establecido la necesidad de darle a la universidad un rol diferente, en torno a la producción y a la transmisión de conocimientos, sin dejarse avasallar por las rigideces de las escuelas profesionales y de la burocracia académica ${ }^{6}$.

Según Pérez Lindo ${ }^{7}$, el concepto de Gestión del Conocimiento, aplicado en la universidad involucra por lo menos estas dimensiones: a) una teoría del conocimiento; b) una teoría de la acción; c) una teoría institucional; d) una política del conocimien- to; e) una metodología para transmitir y aplicar los conocimientos. La Gestión del Conocimiento forma parte del proceso por el cual se trata de valorizar el potencial científico y técnico para mejorar nuestras condiciones de vida.

Gestionar el conocimiento implica reconocer que los seres humanos se vinculan con el mundo y con los conocimientos, a partir de su propia interpretación, generando diferentes percepciones y posibilidades de acción ${ }^{8}$. Cada persona es, a su vez, receptora, productora, formadora e intérprete de los conocimientos, expresados a través del lenguaje y de los instrumentos de comunicación social ${ }^{9}$.

Ese carácter interpretativo de los fenómenos del mundo y del conocimiento, propios de cada persona, genera un espacio de diversidad que, bien aprovechado, favorecería; respetando al otro como un legítimo otro y, por ende, con una escucha efectiva ${ }^{10}$, la creatividad y la generación de nuevos conocimientos e innovación.

Se ha clasificado el conocimiento de distintas maneras, de acuerdo con los autores. Se lo ha llamado: científico y práctico, objetivo y procedente de la experiencia, de procedimiento, incorporado, migratorio, incrustado y codificado, etc. Por otra parte, se debe diferenciar el conocimiento tácito del explícito ${ }^{11}$.

El conocimiento tácito es el que se adquiere desde la experiencia. Es un conocimiento que se adquiere en forma insensible, al incorporarse a una organización o cultura diferente. El conocimiento explícito es el que se transmite con un lenguaje formal y sistemático (Tabla 1).

Gopal y Gagnon $(1995)^{13}$, definen a la Gestión del Conocimiento como un modelo de administración de tres etapas: 1.- del conocimiento, 2.- de información y 3.- de aprendizaje (Figura 1).

En su desarrollo, se produce una transformación del conocimiento individual o tácito, sesgado por las características individuales de los modelos mentales de las personas ${ }^{5}$, en un conocimiento explícito, formal y sistemático; que puede ser comunicado y compartido.

Nonaka $(1991,1993,1994)^{14-16}$ y Nonaka \& Takeuchi $(1995)^{12}$ definen 4 modos de conversión del co- 
Tabla 1

Tipos de conocimiento

\begin{tabular}{|l|l|}
\hline Conocimiento tácito (subjetivo) & Conocimiento explícito (objetivo) \\
\hline $\begin{array}{l}\text { Conocimiento a través de la experiencia } \\
\text { (cuerpo) }\end{array}$ & $\begin{array}{l}\text { Conocimiento a través de la racionalidad } \\
\text { (mente) }\end{array}$ \\
\hline Conocimiento simultáneo & Conocimiento secuencial (en el acto) \\
\hline Conocimiento analógico (práctica) & Conocimiento digital (teoría) \\
\hline Fuente: Nonaka $^{12}$ & \\
\hline
\end{tabular}

Figura 1

Modelo de Gopal \& Gagnon

Fuente: Gopal ${ }^{13}$

Descubrimiento del capital intelectual de la organización, con el objetivo de establecer la situación actual del conocimiento (diagnóstico) y de definir la situación deseada.

ADMINISTRACIÓN DEL CONOCIMIENTO

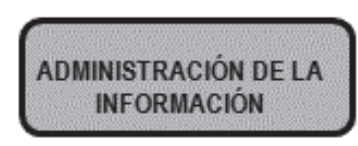

Busca establecer la calidad y las características que debe presentar la información para alcanzar la situación definida en la primera etapa.
Cierra el ciclo de transformación del conocimiento tácito en explícito, al establecer propuestas y prototipos de aprendizaje, asi como formas de cuantificarlo, de manera que el conocimiento y el aprendizaje organizacional se integren en la cultura de la empresa como valores y motive el crecimiento y desarrollo de los individuos

ADMINISTRACIÓN DEL APRENDIZAJE nocimiento: la socialización, la externalización, la combinación y la internalización. Utilizan una palabra: "ba", que es un ideograma kanji, que se originó en los trabajos del filósofo japonés Kitaro Nishida $(1870-1945)^{17}$ y fue incorporada a los procesos de gestión de conocimiento por Nonaka \& Konno $(1998)^{18}$. Cada modo de conversión, se expresa con un "ba". El "ba" origen vinculado a la creación del conocimiento, el "ba" dialogante estimula la reflexión, el diálogo, la lluvia de ideas y propicia el trabajo en equipo. El "ciber-ba" está vinculado a lo tecnológico y el "ba" empírico utiliza simulaciones por escenarios, para ayudar en la internalización del conocimiento (Figura 2).
Expresado en un mapa conceptual, se puede sintetizar como aparece en la figura 3.

El "ciclo" constituye un proceso permanente, que se representa como una espiral (Figura 4).

La espiral ascendente de creación de conocimientos incluye 5 fases: 1.- compartir conocimiento tácito, 2.- crear conceptos, 3.- justificar los conceptos creados, 4.- construir arquetipos y 5.- transferir el nuevo concepto a todos los niveles organizativos. Este modelo ha sido pionero de gran parte de los conceptos que se manejan actualmente y referencia de numerosos trabajos y modelos posteriores. 


\section{Figura 2}

Las cuatro formas de conversión del conocimiento.

Fuente: Nonaka ${ }^{12,14-16,18,19}$

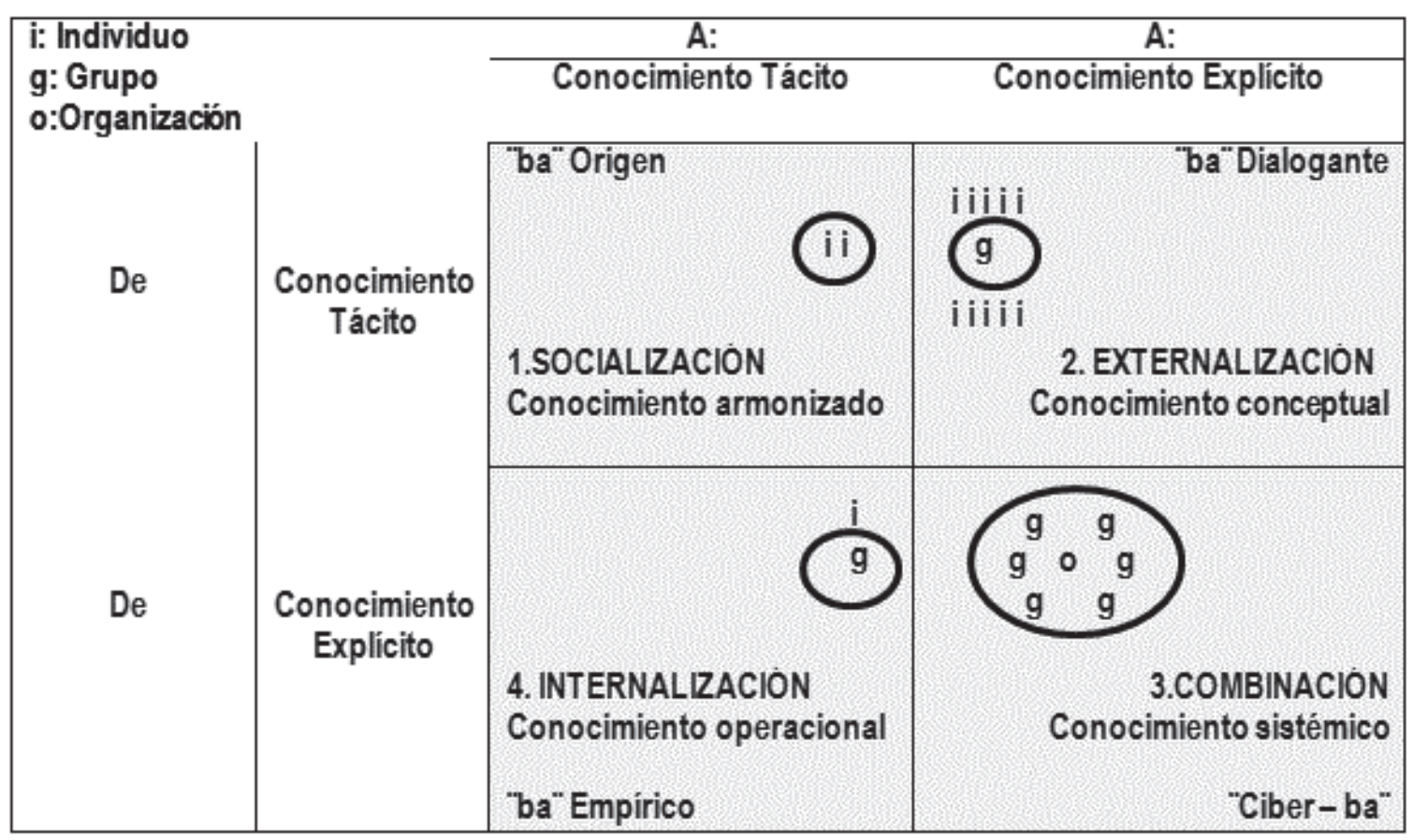

Figura 3

Ciclo de la Gestión del Conocimiento

Fuente: Bueno ${ }^{20}$

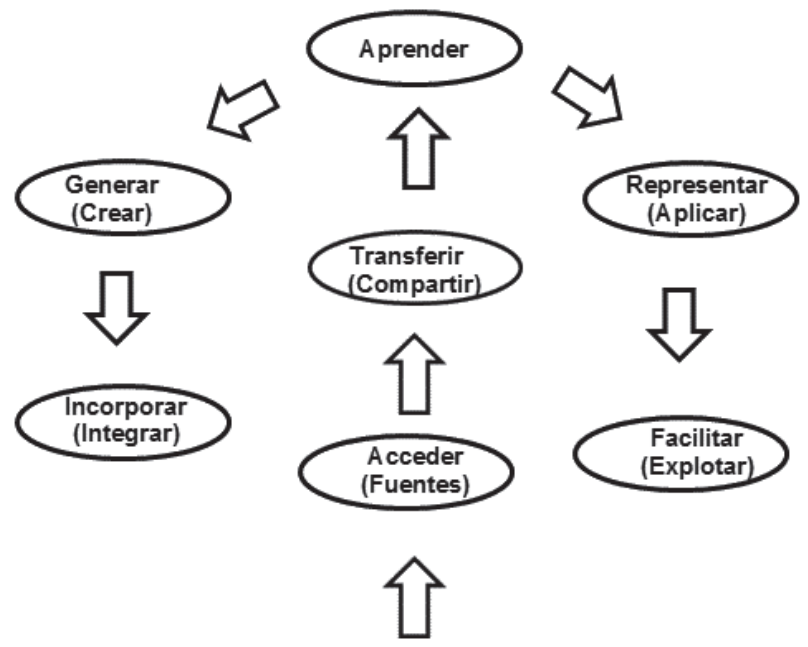




\section{Figura 4}

Espiral de conversión del conocimiento organizativo

$$
\text { Fuente: Nonaka }{ }^{12}
$$

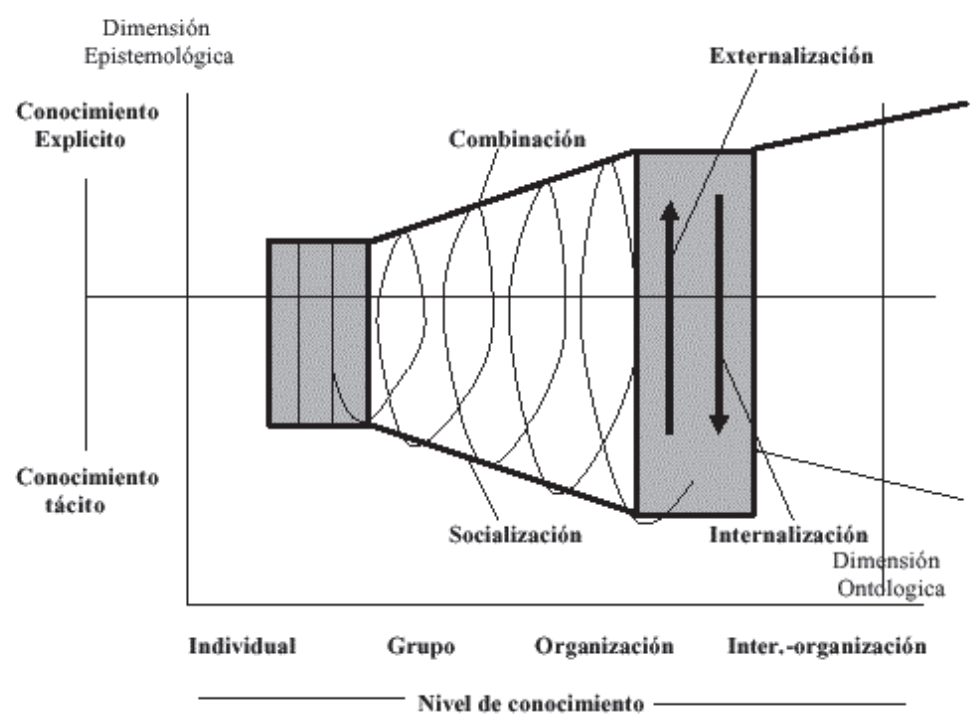

Como define Pérez Lindo ${ }^{2}$, en las universidades la Gestión del Conocimiento tiene su campo de aplicación en la administración académica, en el diseño de los currículos, en sus procesos de reestructuración, en sus propios proyectos de investigación o en programas de cooperación internacional. Es importante lograr una adecuada complementación entre universidad y sociedad, para evitar la paradoja manifestada por el autor al decir que sobran arquitectos y faltan viviendas, sobran médicos y falla el sistema de salud, sobran abogados y el sistema judicial es altamente deficiente.

Es imprescindible enfatizar los procesos de cambio y mejora, los cuales son:

- Reorientar la actividad científica, favoreciendo la labor interdisciplinaria, la resolución de las problemáticas de la sociedad, así como su enriquecimiento cultural, dentro y fuera de los espacios universitarios, lo mural y lo extramural.

- Desarrollar una organización inteligente, evitando la fragmentación en carreras, orientarla a Departamentos, con articulación entre ellos ${ }^{21}$, for- taleciendo la informatización de la universidad, para facilitar la conexión entre todas las áreas, llevando la biblioteca a la acción de enseñar y la investigación a la transferencia de conocimientos. Establecer una política de publicaciones, articular el intercambio del cuerpo de profesores y alumnos hacia el interior y el exterior y generar redes de conocimiento, estableciendo nodos con participación efectiva de los docentes e investigadores. Darle importancia a los programas como una herramienta permanente y una instancia superadora a los esfuerzos individuales.

- Estar abiertos a la reingeniería de procesos para adecuarse a los tiempos de cambio, los adelantos científicos y las nuevas necesidades sociales.

- Establecer una dinámica del currículo de manera inteligente, actualizable durante la etapa formativa para que el graduado posea los conocimientos actualizados para ejercer su profesión.

- Formar a los futuros graduados en las competencias genéricas; abordando el espacio formativo de la inteligencia emocional ${ }^{22}$. 
- Desarrollar una "Biblioteca Total", poniendo a disposición de los estudiantes la mayor cantidad de información disponible, utilizando todas las posibilidades tecnológicas.

Para todo ello, es imprescindible pensar la evolución de las universidades en términos epistemológicos. Fortalecer la reflexión sobre la creación, legitimación y aplicación de los conocimientos en la sociedad ${ }^{23}$.

Para propiciar programas académicos, las tendencias científicas más avanzadas deben alinearse con las demandas de la sociedad, dando lugar a un amplio espacio de acción de responsabilidad social universitaria, posibilitando la simbiosis social con lo científico.

Por lo tanto, una gestión exitosa del conocimiento en los espacios universitarios y de educación superior, en general, requiere:

1. Coherencia institucional. Fines, misiones, funciones y objetivos compartidos por todos los actores.

2. Formulación de programas científicos y académicos, con base en políticas de conocimiento, que incluyan aspectos epistemológicos, pedagógicos, organizacionales y sociales.

3. Apoyar la articulación de los compartimientos institucionales, evitando el aislamiento académico por áreas.

4. Avanzar hacia una adecuada red de información accesible a todos los actores.

5. Establecer lineamientos concretos de articulación con la sociedad, con los actores económicos, el mercado y el Estado.

Pérez Lindo, enfatiza, que el paradigma de la "Gestión del Conocimiento" propone fortalecer las capacidades para participar en la creación y la difusión de los conocimientos para resolver los problemas de la sociedad, para formar especialistas con responsabilidad moral y para extender las fronteras de las ciencias.

\section{Planteamiento del problema}

La enseñanza tradicional en las facultades de medicina, se enfoca en las enfermedades y no en el concepto global de salud y calidad de vida.
Hoy no resulta suficiente haber completado el primer ciclo en la universidad. Es necesario mantener un aprendizaje permanente, sobre todo en las carreras de posgrado, como una manera de mantener actualizado el conocimiento y acompañar los vertiginosos cambios que las demandas sociales presentan.

En las carreras de medicina este aspecto es crucial, por el lapso que media entre la graduación y la finalización de la actividad médica, que va más allá de la jubilación formal, tal como se puede observar en la figura 5.

Hasta ahora las facultades de medicina se han comportado de la siguiente manera:

- Otorgando poco valor al conocimiento tácito.

- Dando prioridad al almacenamiento del conocimiento sobre el flujo del conocimiento.

- Centros de documentación, bases de datos, revistas y páginas Web, sin acercamiento a los equipos de trabajo del Sistema de Salud.

- Teniendo escasa intervención en las normas/ guías de atención y administración de servicios y no participando en las redes asistenciales, incluso de los hospitales universitarios.

En algunos países de Latinoamérica, se ha instalado la acreditación de los médicos cada 5 años (voluntaria). Pero estas evaluaciones, no son suficientes si no se modifican los aspectos curriculares de la enseñanza de la medicina en el pregrado.

Es necesario propiciar una enseñanza más holística de la salud, no solamente en las enfermedades, y sobre todo articular las distintas miradas especializadas, para evitar la fragmentación de la atención médica.

Se propone fortalecer curricularmente los conceptos y contenidos de la Atención Primaria de la Salud, dada la cambiante situación de la salud de las poblaciones, de la composición etaria de la sociedad y del incremento de las "hostilidades" del medio ambiente sobre el ser humano. No se puede desconocer la importancia del envejecimiento poblacional, como factor que explica el incremento de la prevalencia de las enfermedades crónicas. Como ejemplo, la secuencia poblacional de Colombia, expresada en la pirámide fue como aparece en la figura 6. 


\section{Figura 5}

Evolución de la formación en Medicina

Fuente: Depine, S. Elaboración propia

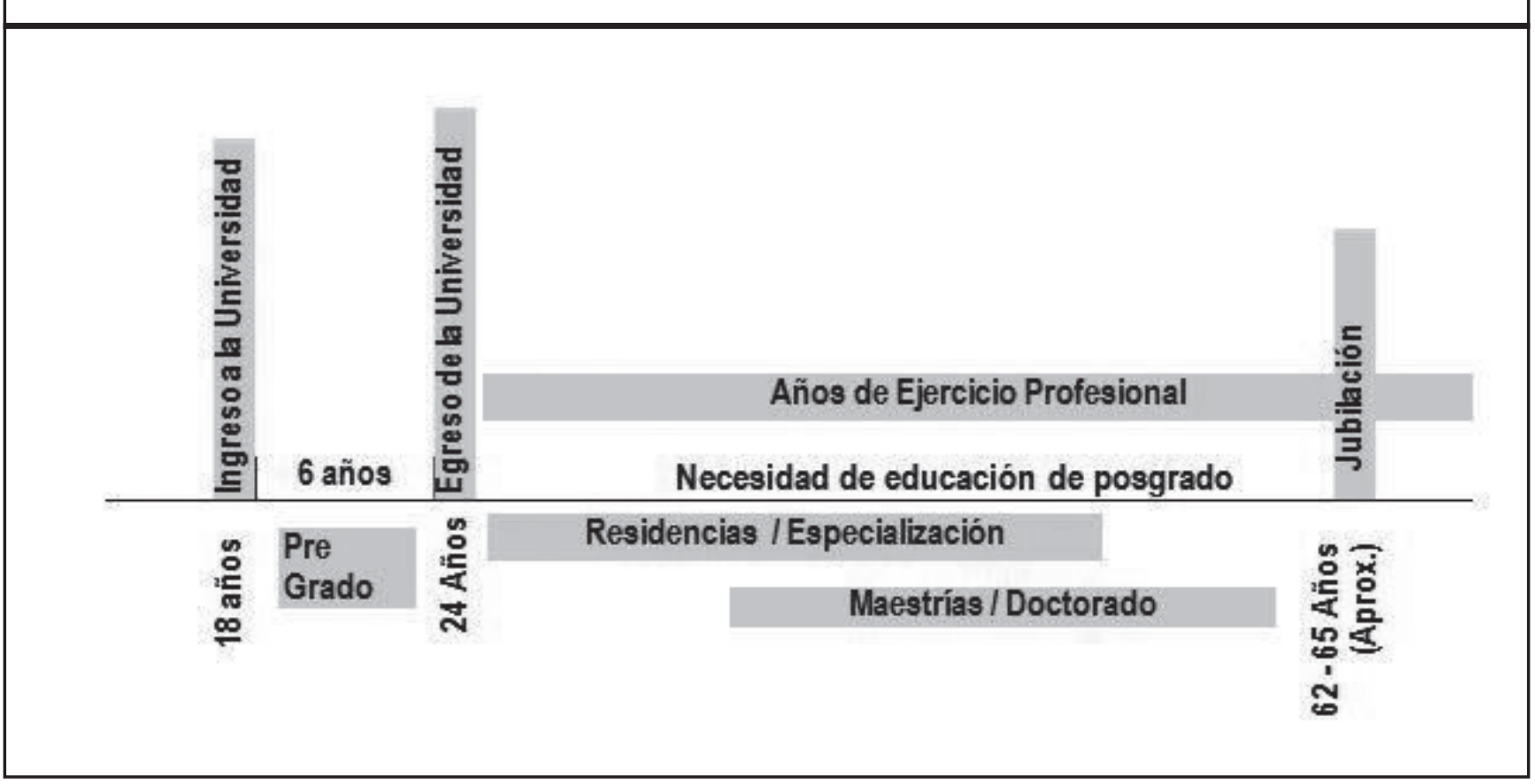

Esta "inversión" de la pirámide, es mucho más evidente en los países más desarrollados, lo cual hace aún más necesaria la reformulación de las políticas de salud, sustentadas en una "novedosa" enseñanza de la medicina en las universidades, paradójicamente regresando a una relación médico-paciente más cercana. Por ejemplo, la pirámide poblacional en Estados Unidos de América muestra una mayor tendencia al envejecimiento poblacional (Figura 7).

A población más envejecida, se corresponde una mayor carga de Enfermedades Crónicas No Trasmisibles (ECNT), mayor demanda de capacidad instalada y mayor presupuesto.

Esta situación, ubica el foco de acción en los espacios universitarios en particular y en los de aprendizaje en general, para transformar la enseñanza universitaria, centrada en la atención de la enfermedad, hacia una nueva conceptualización de la salud, gestionando el conocimiento para formar profesionales del campo de la salud con una visión más holística, con inserción en la labor comunitaria, apoyando las prácticas de mejora de la calidad de vida y de la promoción de hábitos saludables.

Las facultades de medicina, deben ser parte activa de las comunidades del conocimiento y prácticas, fomentando alianzas estratégicas de Gestión del Conocimiento entre los docentes, los decisores de las políticas sanitarias, los proveedores de servicios, las organizaciones intermedias de la sociedad, actuando en los espacios de ciudadanía, los investigadores y los propios pacientes, siendo prioritaria tal acción en las ECNT.

\section{Objetivos}

El objetivo de este proyecto, es impulsar un modelo de Gestión del Conocimiento para incorporar en el currículo universitario de las facultades de medicina, una nueva concepción bio-psico-social-económica del proceso salud-enfermedad-atención-rehabilita- 


\section{Figura 6}

Modificación de la pirámide poblacional a través de los años en Colombia Fuente: Departamento Administrativo Nacional de Estadística (DANE)

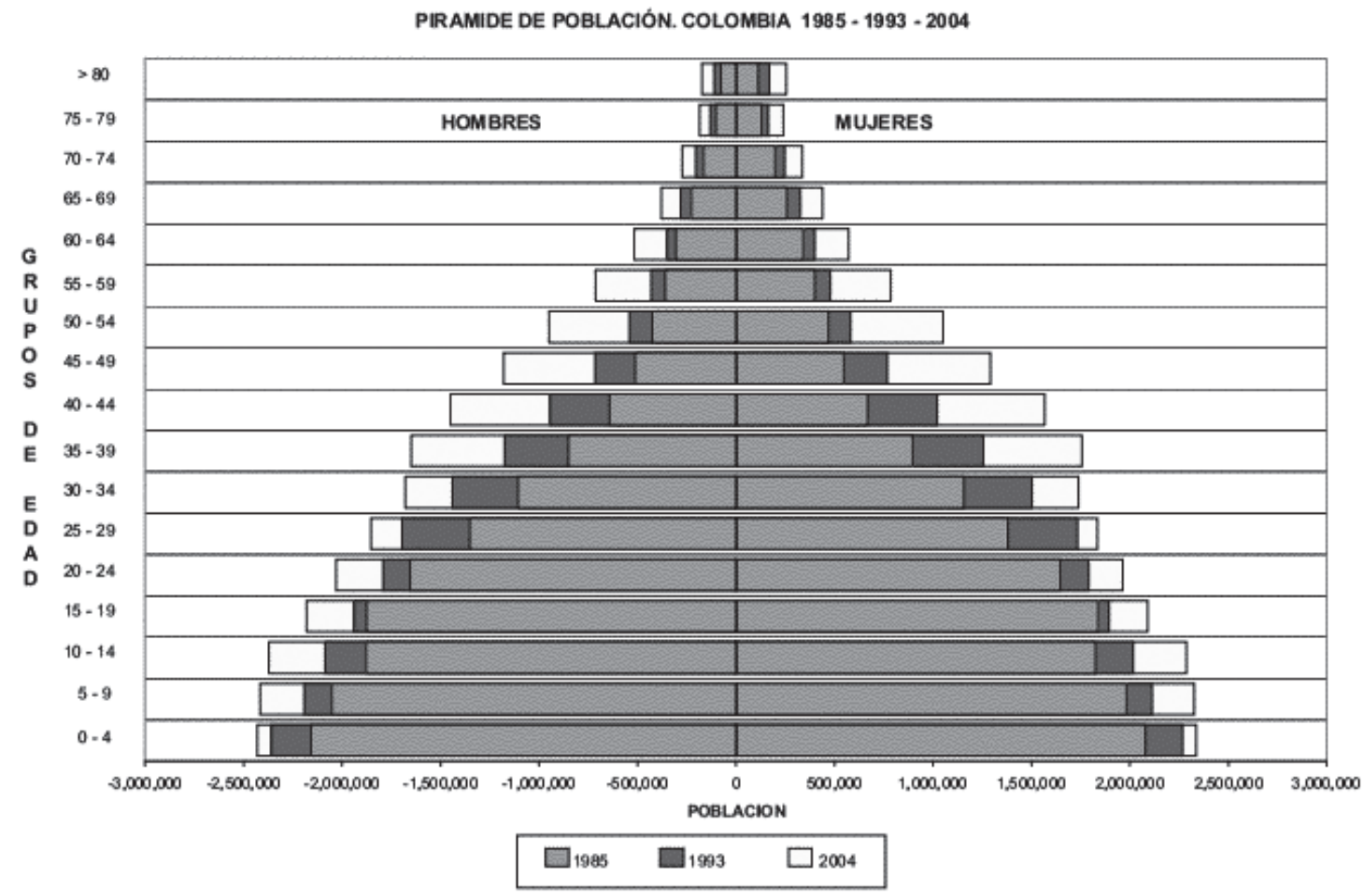

Piramide de población. Colombia 2012

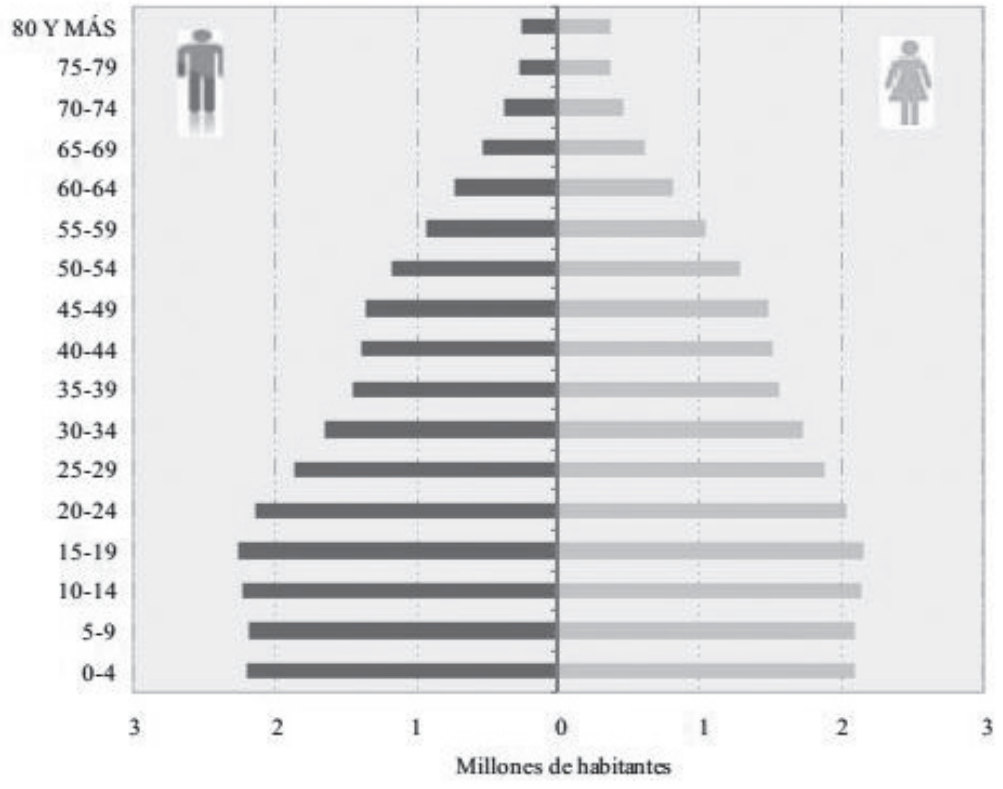




\section{Figura 7}

Pirámide poblacional en Estados Unidos de América

Fuente: U.S. Census Boreau

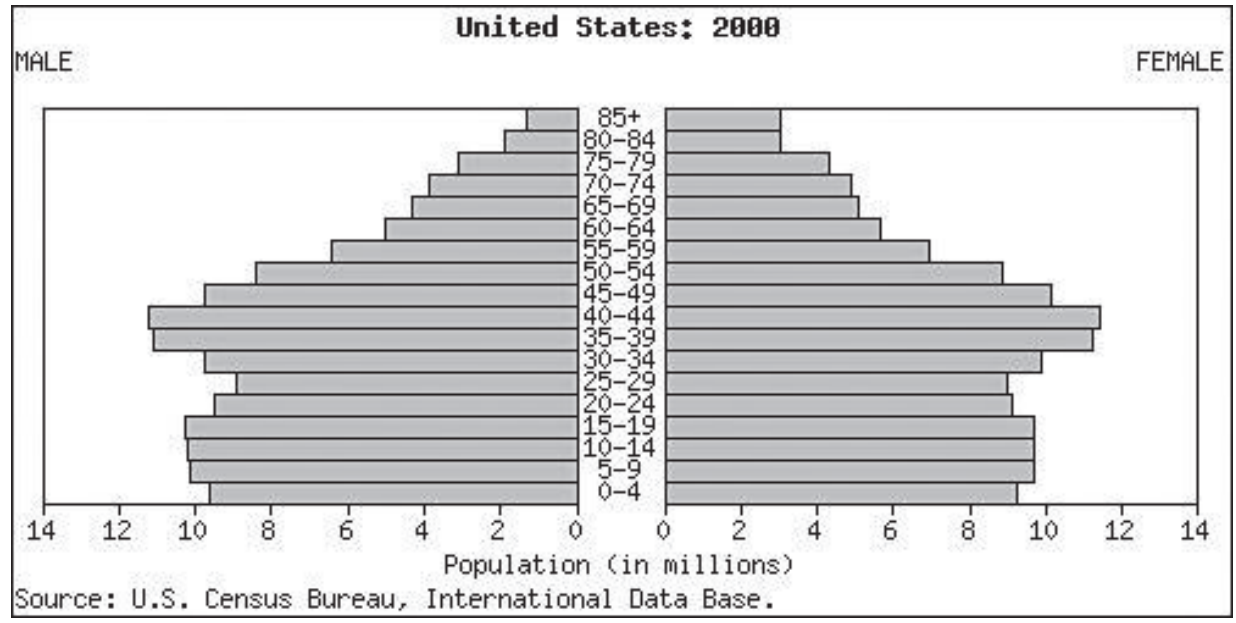

ción-salud, fortaleciendo principalmente los componentes de promoción, prevención y control de las ECNT, como un desafío mundial para el Siglo XXI.

\section{Justificación}

En la actualidad, las ECNT, generan una creciente demanda de tratamientos permanentes de alto impacto tecnológico y de recursos para implementarlos.

Existe un consenso generalizado en darles una ubicación preferencial entre los graves problemas de salud pública a resolver en el corto plazo.

Algunos países pusieron énfasis en políticas públicas saludables y en el control de las enfermedades crónicas, en forma aislada e independiente ${ }^{24-26}$. Sin embargo, en los espacios universitarios no ha habido una sistematización de la Gestión del Conocimiento en esos temas, salvo algunas excepciones ${ }^{27}$.

Como una consecuencia de este impacto, se realizó la Cumbre Mundial sobre Enfermedades No Contagiosas, en septiembre de 2011, a la que asistieron los jefes de Estado para discutir un plan de acción mundial de control de dichas enfermedades. Dicho plan de acción, fue reflejado en la Declaración Política de la Reunión de Alto Nivel de la Asamblea General sobre la Prevención y el Control de las Enfermedades No Contagiosas (Naciones Unidas, 2011), que reconoce que estas patologías suponen un desafío de "proporciones epidémicas" con grave impacto en el desarrollo socio-económico de los países y solicita, de manera urgente, una acción global de los gobiernos y de la sociedad, prestando especial atención a las estrategias de prevención y promoción de la sa$\operatorname{lud}^{28}$.

Su texto reconoce la necesidad de un cambio de paradigma para la enseñanza y el abordaje de los actuales desafíos que se plantean en la salud mundial, dado que persiste la elevada mortalidad por enfermedades cardiovasculares y además la insuficiencia renal y la diabetes mellitus, su principal causa etiológica, se ubican en el rango de epidemia.

Cada vez es mayor la cantidad de personas que necesitan tratamiento mediante diálisis o trasplante, con elevados costos globales que impactan negativamente en las economías de los países, haciéndolos, en muchos casos, imposibles de soportar.

Existe una gran cantidad de personas asintomáticas que presentan enfermedad renal oculta, los cuales 
detectados y tratados precozmente pueden lograr la remisión, retardar la progresión de su enfermedad renal y la reducción de los acontecimientos cardiovasculares, con un uso mucho más eficiente de los recursos necesarios.

Sin embargo, es habitual que; por los déficit formativos en los programas de pregrado y aún en las especializaciones, no se detecten precozmente etapas iniciales de enfermedad, que en el caso de la enfermedad renal, sin diagnóstico y control adecuado, evolucionarán hacia la falla permanente de su función renal, enfermedad cardiovascular y muerte prematura.

Estos pacientes generan enormes gastos de los sistemas financiadores y de los prestadores, deteriorando fuertemente las economías familiares, generando gastos evitables con una correcta vigilancia y control epidemiológico precoz, producto de una buena formación médica en promoción de la salud y prevención de las enfermedades.

\section{Viabilidad del proyecto}

Por todo lo señalado precedentemente, están dadas las condiciones ideales para plasmar un proyecto curricular en las facultades de medicina, basado en estrategias de atención primaria de la salud, con foco en la promoción de la salud y la prevención de las enfermedades crónicas, sin dejar de lado los componentes de la atención de las enfermedades, la rehabilitación y las técnicas de remplazo de las funciones deterioradas.

\section{Limitaciones del proyecto}

Implica un cambio paradigmático, lo cual no suele ser sencillo. La modificación de los currículos de estudio tampoco resulta fácil de lograr.

\section{Líneas de acción e implementación del proyecto}

En este proyecto se hará foco en la Gestión del Conocimiento en las facultades de medicina, generando los mecanismos curriculares y actividades, que posibiliten el aprovechamiento de los conocimien- tos tácito y explícito, para transformar este último en una herramienta disponible y accesible para los estudiantes, tanto en el pregrado como en los posgrados universitarios.

Dada la importancia y el impacto de las ECNT, transformar el conocimiento tácito existente en conocimiento explícito, potenciando a su vez este último como producto final de lo académico y científico. Todo esto para:

- Hacerlo más accesible.

- A partir de él transformarlo en capacidad para la acción.

- Trasladarlo a los pacientes para fomentar su autocuidado.

En relación con la importancia dada al conocimiento tácito, el artículo h de la Declaración de Naciones Unidas, manifiesta: "Reconocer en mayor medida el potencial y la contribución de los conocimientos tradicionales y locales y, a ese respecto, respetar y preservar, de conformidad con las capacidades, las prioridades, la legislación y las circunstancias nacionales, los conocimientos y la utilización segura y eficaz de la medicina tradicional, sus tratamientos y prácticas, basándose, según proceda, en las circunstancias imperantes en cada país".

Para llevarlo a cabo, se propone:

- Incorporar tecnologías de información y comunicación (TICs) a los procesos de construcción de conocimientos (enseñanza-aprendizaje) para desarrollar el análisis de información y la captura de conocimientos.

- Dar visibilidad y articular los mecanismos para que las comunidades de conocimientos y de prácticas del Sector Salud, sean mediadas por saberes elaborados o populares, se constituyan en verdaderos espacios de aprendizaje, desde lo extramural del sector académico estricto, potenciando la extensión universitaria.

- Generar alianzas estratégicas para desarrollar la Gestión del Conocimiento, realizando cogestión curricular de contenidos y de plataformas de las TICs, al servicio de la formación de recursos humanos para la salud. 
- Fortalecer la participación de los alumnos en los espacios asistenciales comunitarios; de preferencia en los Centros de Atención Primaria, en forma temprana, para apoyar la integración docente-asistencial.

- Establecer líneas de investigación académica, sobre los sistemas y servicios de salud potenciando la vinculación docente - alumno con la problemática socio sanitaria.

- Incorporar la conceptualización de la Gestión del Conocimiento (de lo tácito a lo explícito y viceversa) a los diseños curriculares, generando procesos educativos dinámicos que incluyan socialización de aprendizajes, trabajo en equipo, utilización y creación de redes de información, estableciendo el uso de los portales de conocimiento (e-learning).

- Adecuar los centros de información/bibliotecas a las nuevas propuestas.

- Dar participación activa a la universidad para la organización de la información y generar bases de conocimientos, que den sustento a las prácticas médicas de acuerdo con la evidencia científica disponible. Tomar ejemplo de las universidades que lo hacen actualmente, por ejemplo el Centre for Evidence-Based Medicine, Department of Primary Health Care, de la Universidad de Oxford (RU).

- Transformar los archivos de documentos en algo dinámico generando mapas de conocimientos.

- Reforzar la noción de que la universidad gerencia el conocimiento y que el mismo se transforma en significativo cuando el estudiante se hace protagonista autogestionarlo el conocimiento.

- Potenciar en el docente un rol facilitador y transformador, proveyendo valor agregado al proceso de autogestión del conocimiento.

- Desarrollar en el entorno universitario una estructura especializada en Gestión de Conocimientos y Arquitectura de Información, que pueda seleccionar, evaluar, sintetizar y eventualmente comentar las publicaciones que aporten los mejores contenidos prácticos, a través de fuentes de información secundarias.
- Apoyar a los alumnos en el diseño e interpretación de trabajos científicos, metaanálisis, en el uso de buscadores especializados.

- Propiciar, entre alumnos y profesores, espacios de aprendizaje como un sistema experto y organización de la información en comunidades de conocimiento.

- Utilizar y diseñar instrumentos y contenidos de aprendizaje a distancia con TICs.

- Habituar a los alumnos y graduados en el uso de las herramientas de diseminación de conocimientos, participación en redes virtuales.

- Gestionar el conocimiento de tal forma que posibilite que el alumno y el graduado en los programas de extensión universitaria, comprendan y participen en la administración de los recursos en salud, en el entendimiento que el médico es el primer gestor de recursos del sistema de salud.

- Transformarse en un aliado imprescindible de los organismos reguladores y generadores de políticas sanitarias en cada uno de los países.

- Por último, realizar el proceso dinámico de actualización de los currículos instrumentando la Gestión de los Conocimientos, ya sea en los aspectos clínicos o en el mantenimiento y fortalecimiento del capital intelectual necesario para alcanzar los objetivos propuestos en los Programas de Salud Pública de control de la ECNT; participando activamente en el diseño y generando capacitaciones extracurriculares y de extensión, para garantizar la correcta implementación de las funciones esenciales de la salud pública en los programas con prioridades en la salud cardiovascular, renal y endocrinometabólica de la sociedad.

\section{Resultados esperados}

Una formación más integral de los graduados en medicina, permitirá:

- Menor gasto global de los sistemas de salud a corto, mediano y largo plazos.

- Menor gasto global de las familias y de la comunidad. 
- Menor morbimortalidad global y mejor calidad de vida.

- Ahorros significativos en el gasto en salud, tanto para los organismos financiadores, los prestadores y las familias.

- Facilitación en el uso e interpretación de indicadores epidemiológicos, estadísticos y curvas actuariales.
- Involucramiento de los graduados en el establecimiento de medidas correctivas en el seguimiento de los pacientes, la contención del gasto y correctas previsiones presupuestarias a futuro.

\section{Conflicto de interés}

El autor declara no tener ningún conflicto de interés.

\section{Referencias bibliográficas}

1. Geertz, C. Conocimiento local. Ensayos sobre interpretación de las culturas. 1a ed. Barcelona: Paidós; 1994.

2. Pérez Lindo A, Ruiz Moreno L, Varela CG, Camós CT, Trottini AM, Luján Burke M. Gestión del conocimiento. Un nuevo enfoque aplicable a las organizaciones y a la universidad. 1a ed. Buenos Aires: Norma; 2005.

3. Drucker P. Las nuevas realidades. 2a ed. Buenos Aires: Sudamericana; 1990.

4. Clark B. El sistema de educación superior. Una visión comparativa de la organización académica. 1a ed. México: Nueva Imagen; 1991.

5. Senge P. La quinta disciplina. 4a ed. Barcelona: Granica; 1996.

6. García Guadilla C. Educación superior comparada. El protagonismo de la internacionalización. 1a ed. Caracas, Venezuela: IESALC-UNESCO / CENDES / bid \& co. Editor; 2010.

7. Pérez Lindo A. Fundamentos de la gestión del conocimiento en la universidad. Gestión Universitaria 2011; 03 (02). [Recuperado el 16 de febrero de 2014], disponible en http://www.gestuniv.com.ar/gu_08/v3n2a1.htm.

8. Echeverría R. Ontología del Lenguaje. 5a ed. Santiago de Chile: Granica; 1998.

9. Pérez Lindo A, compilador. El concepto de realidad: teorías y mutaciones. 1a ed. Buenos Aires: Proyecto Editorial; 2003.

10. Maturana RH. La objetividad. Un argumento para obligar. 2a ed. Santiago de Chile: J.C. Saez; 2007.

11. Polanyi M. The Tacit Dimension. 1a ed. London: Routledge \& Kegan Paul; 1966.

12. Nonaka I. Takeuchi H. La organización creadora de conocimiento: Cómo las compañías japonesas crean la dinámica de la innovación. Trad. Martín Hernández Kocka. 1a ed. México: Oxford University Press; 1999.

13. Gopal C. Gagnon J. Knowledge, Information, Learning and the IS Manager. Computerworld (Leadership Series) 1995; 1(5): 1-7.

14. Nonaka I. The Knowledge-Creating Company. Harvard Business Review 1991. November-December: 96-104.

15. Nonaka I. On a Knowledge Creating Organization. Economía \& Management 1994; 3 (Mayo): 31-48.

16. Nonaka I. A Dynamic Theory of Organizational Knowledge Creation. Organization Science. 1994; 5 (1):14-37.

17. Nishida K. An Inquiry into the Good. (M. Abe, \& C. Ives, Trads.) 1a ed. New Haven, Connecticut: Yale University Press 1992; 40 (3): 40-54.

18. Nonaka I. Konno N. The concept of Ba: Building a Foundation for Knowledge Creation. California Management Review 1998. 40 (3): 40-54.

19. Nonaka I. Reinmoeller P. Senoo D. El ART del conocimiento: sistemas para rentabilizar el conocimiento del mercado. Harvard Deusto Business Review 1999. 92: 621 
20. Bueno E. El capital intangible como clave estratégica en la competencia actual. Boletín de Estudios Económicos 1998; 164 (53): 207-229.

21. García de Fanelli A. Profesión Académica en la Argentina: Carrera e Incentivos a los docentes en las Universidades Nacionales. 1a ed. Buenos Aires: CEDES; 2009.

22. Goleman D. Boyatzis R, Mc Kee A. El líder resonante crea más. 3a ed. Buenos Aires: Sudamericana/Plaza Janes; 2003.

23. Pérez Lindo A. La universidad futura: de la utopía al realismo posibilista. En: Red Argentina de Postgrado en Educación Superior. Ensayos e investigaciones sobre la problemática universitaria. San Luis: Universidad Nacional de San Luis; 1997. Cap. I, p.15-28.

24. Depine S. The role of government and competing priorities in minority populations and developing nations. Ethnicity \& Disease 2009; 19(S1):73-79.

25. Depine S. Burgos CR. Renal Health Models in Latin America: Development of National Programs of Renal Health. Renal Failure, 2006; 28: 649-664.

26. Burgos CR. Depine S. Sustainable and tenable renal health model: A Latin American proposal of classification, programming, and evaluation. Kidney Int, 2005; 68 (97): S23-S30.

27. Burgos CR. Depine S. Systematic approach for the management of chronic kidney disease: moving beyond chronic kidney disease classification. Curr Opin Nephrol Hypertens. 2010;19 (2):208-13.

28. Asamblea General de las Naciones Unidas. Declaración Política de la Reunión de Alto Nivel de la Asamblea General sobre la Prevención y el Control de las Enfermedades No Transmisible; 16 de Septiembre de 2011. http://www.un.org:http://www.un.org/es/comun/docs/?symbol=A/66/L.1 